\title{
VLBI observations of GRB afterglows
}

\author{
G.B. Taylor ${ }^{1}$, A.J. Beasley ${ }^{1}$, D.A. Frail ${ }^{1}$, S.R. Kulkarni ${ }^{2}$, and J.E. Reynolds ${ }^{3}$ \\ 1 National Radio Astronomy Observatory (NRAO), Socorro, NM 87801, U.S.A. \\ e-mail: gtaylor@nrao.edu \\ 2 Division of Physics, Mathematics, and Astronomy 105-24, California Institute of Technology, Pasadena, CA 91125, U.S.A. \\ 3 Australia Telescope National Facility, CSIRO, Epping 2121, Australia
}

Received December 29, 1998; accepted March 30, 1999

\begin{abstract}
Once the afterglow from a GRB has been detected in the radio, Very Long Baseline Interferometry (VLBI) observations can provide a resolution of 1 milliarcsecond or better and absolute astrometry at a similar level. We will review the VLBI programs carried out to date which include observations of G970508, G980329, G980425, and G980703. All of these objects are found to be very compact (size $<1$ mas) even months after the burst. This is consistent with the predictions of fireball models. Our ultimate goal is a direct measurement of the morphology and expansion of a GRB afterglow.
\end{abstract}

Key words: gamma-ray bursts - technique: interferometry astrometry

\section{Introduction}

Four out of five radio afterglows (see Table 1) have been observed with VLBI techniques. In this paper we review what has been learned so far, and discuss future applications.

\section{VLBI observations of radio afterglows}

G970508: A first epoch VLBA observation was obtained just 8 days after the gamma-ray burst, and within 48 hours of the VLA discovery of a radio counterpart (Frail et al. 1997a). An additional 8 epochs were obtained until $\sim 120$ days after the burst when the radio afterglow faded below $0.3 \mathrm{mJy}$, the nominal sensitivity limit. From this data we derived a position accurate to better than 0.1 mas, placed a $3 \sigma$ limit on the proper motion of less than 5 mas $\mathrm{y}^{-1}$ and placed a limit on the parallax of less than 0.3 mas corresponding to a distance limit of $>3 \mathrm{kpc}$

Send offprint requests to: G.B. Taylor
Table 1. Radio afterglows as of 1998 November

\begin{tabular}{|c|c|c|c|}
\hline \multirow[b]{2}{*}{ GRB } & \multicolumn{3}{|c|}{$8 \mathrm{GHz}$} \\
\hline & $\begin{array}{l}S_{\text {peak }} \\
\text { (mJy) }\end{array}$ & $\begin{array}{c}\tau_{\text {peak }} \\
\text { (days) }\end{array}$ & $\begin{array}{c}\text { VLBI } \\
\text { detection }\end{array}$ \\
\hline G970508 & 0.63 & 60 & Yes \\
\hline G980329 & 0.35 & 40 & No \\
\hline G980425 & 50.0 & 11 & $? ? ?$ \\
\hline G980519 ${ }^{a}$ & 0.20 & 30 & - \\
\hline G980703 & 1.0 & 10 & Yes \\
\hline
\end{tabular}

a No VLBI observations were attempted for G980519.

(Taylor et al. 1997; Taylor et al. 1998). For all epochs the limit on the angular size was less than 0.5 mas $(<4 \mathrm{pc})$.

G980329: We observed, but did not detect, G980329, because variability drove it below $0.2 \mathrm{mJy}$ on the day we observed. The radio afterglow from this source peaked below $0.5 \mathrm{mJy}$ so we did not attempt further observations.

G980425: Associated with SN1998bw in the galaxy ESO 184-G82 at $z=0.0083$, G980425 was studied with the ATCA during the first 60 days of its outburst (Kulkarni et al. 1998) and both scintillation and brightness temperature arguments predict a size of 0.1 mas on day 16 . This time is near the peak in the radio light curve of $50 \mathrm{mJy}$ at $8.4 \mathrm{GHz}$. Unfortunately, only limited VLBI observations were feasible for this far southern declination $\left(-53^{\circ}\right)$ source. On May 10 we observed G980425 at $22 \mathrm{GHz}$ with the VLBA antenna at Mauna Kea and with the 70 meter antenna of the Deep Space Network at Tidbinbilla. The radio source was not detected above a $3 \sigma$ limit of $30 \mathrm{mJy}$. On August 19 we observed with the same two telescopes but at $8.4 \mathrm{GHz}$ and employing phase-referencing to increase the coherence time. No source was detected above a $3 \sigma$ limit of $2.5 \mathrm{mJy}$. An $8.4 \mathrm{GHz}$ observation by a southern hemisphere network of radio telescopes on May 9 th is still being analyzed. 


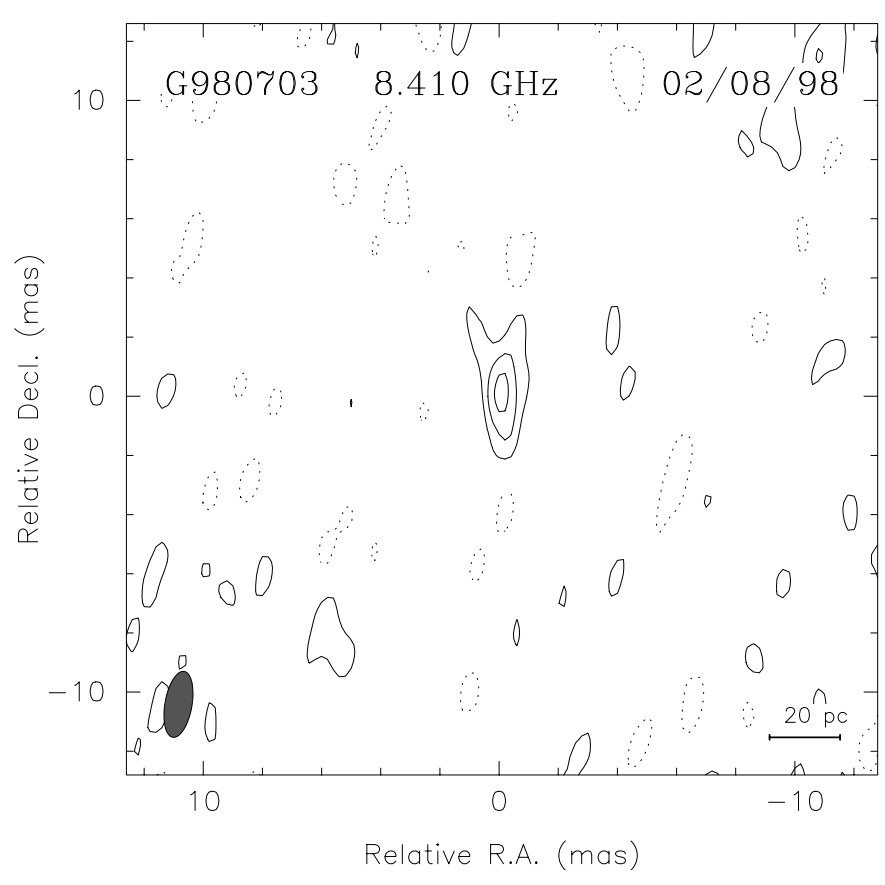

Fig. 1. VLBA observation of G980703 at $8.4 \mathrm{GHz}$ for 5 hours. The synthesized beam is drawn in the lower left-hand corner of the plot and has dimensions: $2.2 \times 0.92$ mas in position angle $-10^{\circ}$. Contours are drawn at $-0.1,0.1,0.2$, and $0.3 \mathrm{mJy} / \mathrm{beam}$

G980519: No VLBI observation was attempted of this very faint radio afterglow.

G980703: The radio afterglow from G980703 reached $1 \mathrm{mJy}$ in the first two weeks after the burst. We detected G980703 at a level of 0.5 mJy 30 days after the burst, when it was already in decline (Fig. 1). We place a limit on the angular size of G980703 of $<0.3$ mas, and derive a position of $\alpha(\mathrm{J} 2000)=23^{\mathrm{h}} 59^{\mathrm{m}} 06.6661, \delta(\mathrm{J} 2000)=8^{\circ} 35^{\prime} 07^{\prime \prime} \cdot 0939$ with an uncertainty of 0.0007 arcsec in each coordinate.

\section{What we can learn from VLBI}

Two weeks after the explosion the size of G970508, estimated from the observed scintillation (Frail et al. 1997b), was 0.003 mas. G970508 reached a peak of $0.7 \mathrm{mJy}$ (with considerable variability) 60 days after the gamma-ray burst. At this time we can estimate a size of $\sim 0.02$ mas from equipartition arguments, unresolvable by VLBI observations (see Table 2). A similar GRB at a redshift of 0.2 would be $\sim 40 \mathrm{mJy}$, and have a size of 0.07 mas 60 days after the burst. With an easily achievable dynamic range image of 300:1, such a burst would be resolved by the VLBA. Unfortunately, if GRBs are homogeneously distributed out to $z=4$, or trace the star formation in the universe, then our chances of observing a GRB at $z<0.2$ are less than 1 in $10^{4}$ (assuming $\Omega_{0}=0$ ). Belying that simple picture, one GRB, G980425, has already been found at $z=0.0083$. This has been explained as either a separate class of GRBs (Bloom et al. 1998), or the result of
Table 2. VLBI limits on expansion

\begin{tabular}{lccccc}
\hline GRB & $z$ & $\begin{array}{c}t_{\mathrm{VLBI}} \\
\text { (days) }\end{array}$ & $\begin{array}{c}S_{\mathrm{t}} \\
(\mathrm{mJy})\end{array}$ & $\begin{array}{c}\theta_{\mathrm{eq}} \\
(\mathrm{mas})\end{array}$ & $\begin{array}{c}\theta_{\mathrm{VLBI}} \\
(\mathrm{mas})\end{array}$ \\
\hline \hline G970508 & 0.835 & 60 & 0.63 & 0.02 & $<0.3$ \\
$\mathrm{G} 980425$ & 0.008 & 14 & 35.0 & 0.08 & $<? ?$ \\
G980703 & 0.965 & 32 & 0.58 & 0.01 & $<0.3$ \\
\hline \hline
\end{tabular}

beaming such that only a small fraction of distant GRBs are detected (Wang \& Wheeler 1998). By day 60 the predicted size of G980425 was 0.4 mas. Such an object in the northern sky would be easy to study with the VLBA.

Granot et al. (1998) have recently computed images of what the relativistically expanding fireball showing a disklike, limb brightened geometry. If GRBs are substantially beamed (as is often invoked to reduce the energy requirements), then we expect substantial deviations from these predictions. Estimates of the rate of expansion also differ greatly (Waxman 1997 vs. Sari et al. 1998). By resolving the explosion the VLBA could play a crucial role in understanding the physics of these energetic events.

Combined VLA and VLBA observations can rule out significant gravitational lensing on scales of 10 mas 300 arcsec in G970508 and G980703. This covers the range of lenses discussed in the literature, and rules out any substantial flux magnification of the gamma-ray burst by gravitational lensing.

The location of the GRB within the host galaxy is also a subject of interest. VLBA observations can pinpoint the location to within 1 mas (or a few parsecs at $z \sim 1$ ). Future optical or IR instruments capable of high resolution and absolute astrometry could use the VLBA position to shed light on the environment of the progenitor object.

Acknowledgements. The NRAO is a facility of the National Science Foundation operated under cooperative agreement by Associated Universities, Inc.

\section{References}

Bloom J.S., Kulkarni S.R., Harrison F., Prince T., Phinney E.S., Frail D.A., 1998, ApJL 506, L105

Frail D.A., et al., 1997a, IAUC, No. 6662

Frail D.A., Kulkarni S.R., Nicastro L., Feroci M., Taylor G.B., 1997b, Nat 389, 261

Granot J., Piran T., Sari R., 1998, (astro-ph/9808007)

Kulkarni S.R., et al., 1998, Nat 395, 663

Sari R., Piran T., Narayan R., 1998, ApJ 497, L17

Taylor G.B., Frail D.A., Beasley A.J., Kulkarni S.R., 1997, Nat 389, 263

Taylor G.B., Beasley A.J., Frail D.A., Kulkarni S.R., 1998, in: Gamma-Ray Bursts, 3th Huntsville Symposium, Meegan C.A., Preece R.D., Koshut T.M. (eds.). AIP: New York, p. 571

Waxman E., 1997, ApJ 489, L33

Wang L., Wheeler J.C., 1998, ApJL 504, L87 